\title{
In Response to Guidewire Entrapped in the Right Ventricle: A Rare Complication of Hemodialysis Catheter Insertion
}

\author{
Rajnish Kumar
}

\begin{abstract}
Verma et al. meticulously prevented the further complications once they recognize the entrapped guidewire in the right ventricle. Authors were very correct to mention overzealous insertion of the guidewire without watching the monitor, which may lead to such complications; however, every time monitor is not available for assistance. The Seldinger technique is routinely used for inserting central venous (CV) and hemodialysis (HD) catheters; however, both are different entities and their designs and lengths including of their guidewires vary. During guidewire insertions, simply adhering to the safe length may prevent or minimize many guidewire-related complications.

Keywords: Guidewire, Guidewire complication, Hemodialysis.

Indian Journal of Critical Care Medicine (2020): 10.5005/jp-journals-10071-23486
\end{abstract}

Sir,

Verma et al. meticulously prevented the further complications once they recognize the entrapped guidewire in the right ventricle. Authors were very correct to mention overzealous insertion of the guidewire without watching the monitor, which may lead to such complications; however, every time monitor is not available for assistance. Definitely, both central venous (CV) and hemodialysis (HD) catheters are routinely inserted using the Seldinger technique (ST), but both are different entities and their designs and lengths including of their guidewires vary. The length of the guidewire supplied with the most of the commercially available CVC sets in adult is about $45 \mathrm{~cm}$ while those of nontunneled HDC sets is about $70 \mathrm{~cm}$ for smooth insertion of the catheter over guidewire. Its helps us in better understanding the situation, if Verma et al. describe the type and make of the HD catheter they had selected for insertion. ${ }^{1}$

The insertion of an excessive length of guidewire during central venous catheterization is the culprit for most of the guidewire-related complications. Many reasons stand behind this faulty practice including not knowing its dangerous implications, fear of losing vascular access, absence of marks on the guidewire, and concerns over contamination of the proximal end of the wire. ${ }^{2}$ The practice of introducing only the length of guidewire necessary to ensure vascular access (i.e., "safe length") is one way to reduce or eliminate the risk of entrapment resulting from looping or knotting of the guidewire. Most guidewires come with marking; before inserting the guidewire, required length is calculated using the Pere formula, landmark or EC-ECG, or keeping in mind the simple fact that the upper limit of safe guidewire insertion in an adult patient from venous puncture point is about $60-70 \mathrm{~cm}$, which might help avoid these complications. Rufener et al. evaluated 30 commonly used central venous catheter kits and found a mismatch between guidewire and catheter length and a general lack of guidewire markings and standardization of the evaluated kits. To prevent such type of complications, manufactures should standardize guidewire distance markings and prepare guidewires specific for each kit, while operator should choose a catheter kit without guidewire length discrepancies and most importantly adhere to the safe length during insertion. ${ }^{3}$
Department of Anesthesia and Critical Care, Medi-Heart Hospital, Patna, Bihar, India

Corresponding Author: Rajnish Kumar, Department of Anesthesia and Critical Care, Medi-Heart Hospital, Patna, Bihar, India, Phone: +91 8084510290, e-mail: rajnishsomya@gmail.com

How to cite this article: Kumar R. In Response to Guidewire Entrapped in the Right Ventricle: A Rare Complication of Hemodialysis Catheter Insertion. Indian J Crit Care Med 2020;24(7):606-607.

Source of support: Nil

Conflict of interest: None

However, in spite of all precautions, unfortunately if guidewire is entrapped during central venous cannulation, Unnikrishnan et al. have devised a very simple traction-less technique for retrieval of stuck guidewire. In their technique, the guidewire is firmly held by three (middle, ring, and little) fingers against the palm while applying a forward force on the guidewire with the thumb and index finger of the same hand. This maneuver will straighten the J-tip without needing a traction force on the guidewire for its retrieval. This technique not only avoids the guidewire-related complications but also preserves the "memory" of the J-tip for its reinsertion and avoids relocation of the vein. ${ }^{4}$

In summary, the ST is routinely used for inserting CV and HD catheters; however, both are different entities and their designs and lengths including of their guidewires vary. During guidewire insertions, simply adhering to the safe length may prevent or minimize many guidewire-related complications.

\section{References}

1. Verma A, Chitransh V, Jaiswal S, Vishen A, Sheikh WR, Haldar M, et al. Guidewire entrapped in the right ventricle: a rare complication of hemodialysis catheter insertion. Indian J Crit Care Med 2020;24(1):8081. DOI: 10.5005/jp-journals-10071-23334.

2. Khasawneh FA, Smalligan RD. Guidewire-related complications during central venous catheter placement: a case report and review of the literature. Case Rep Crit Care 2011;2011:287261. DOI: $10.1155 / 2011 / 287261$. 
3. Chakraborty A, Donoo FA, Roy Chowdhury S, Saha A, Ganguly S, Rupert E. Guidewire entrapment in a tricuspid valve apparatus. J Cardiothorac Vasc Anesth 2010;24(5):898-899. DOI: 10.1053/j. jvca.2009.10.010.
4. Unnikrishnan KP, Sinha PK, Nalgirkar RS. An alternative and simple technique of guidewire retrieval in a failed Seldinger technique. Anesth Analg 2005;100(3):898-899. DOI: 10.1213/01. ANE.0000146654.99367.DD. 